\title{
Modelagem de viabilidade econômica para sistemas fotovoltaicos de geração distribuída
}

\author{
Economic viability modeling for distributed production photovoltaic systems
}

Eduardo Nadler ${ }^{1}$ (ib) orcid.org/0000-0003-3866-6222

Antonio Perrelli 2,3 (D-orcid.org/0000-0002-0261-6330

${ }^{1}$ Pós-graduação em Energias Solar e Eólica, Escola Politécnica de Pernambuco, Pernambuco, Brasil

2 Pós-graduação em Energias Solar e Eólica, Escola Politécnica de Pernambuco, Pernambuco, Brasil

3 Companhia Hidroelétrica do São Francisco, Pernambuco, Brasil

E-mail do autor principal: Eduardo Nadler eduardonadler@hotmail.com

\section{Resumo}

Sistemas fotovoltaicos de micro e minigeração distribuída destacam-se como uma alternativa para que os consumidores possam utilizar a energia gerada por seus sistemas a fim de suprir suas necessidades de consumo. A vida útil de projetos de geração distribuída é de aproximadamente 25 anos, período estimado para que o sistema produza pelo menos $80 \%$ de sua capacidade inicial. Com o propósito de avaliar a viabilidade econômica de instalação de sistemas fotovoltaicos neste período, comumente são utilizados softwares existentes no mercado. Este artigo tem por finalidade modelar a tomada de decisão do consumidor e aplicar o Método de Monte Carlo para efetuar simulações de risco-retorno.

Palavras-Chave: Geração distribuída; Viabilidade econômica; Modelagem Econômica

\begin{abstract}
Photovoltaic systems of micro and minigeration distributed stand out as an alternative for energy production by the consumers in order to supply their need of consumption. The life span of distributed generation projects is approximately 25 years. This period is an estimation that the system will produce at least $80 \%$ of its initial capacity. Proper softwares are often used in order to evaluate the economic feasibility of photovoltaic systems during this period. This paper aims to model the consumer decision-making process and apply the Monte Carlo Method in order to perform risk-return simulations.
\end{abstract}

Key-words: Distributed generation; Economic viability; Economic Modeling 


\section{Introdução}

No Brasil, atualmente existem três tipos de subsistemas geradores: a geração centralizada que consiste em grandes empreendimentos utilizados para geração de energia em larga escala distante do consumidor; sistema isolado, fruto da inexistência de interligação entre os centros consumidores e submercados, e a geração distribuída.

A geração distribuída pode ser explicada como uma unidade geradora de energia instalada diretamente ou próxima ao consumidor final conectado (Grid-Tie) ou desconectada à rede de distribuição (Off-Grid).

A Resolução Normativa 482/2012 teve por objetivo estabelecer as condições gerais para o acesso de micro e minigeração distribuída aos sistemas de distribuição e compensação de energia elétrica. [1]

A Audiência Pública 037/2017 propôs o debate para aprimoramento da Resolução Normativa. Dentre os resultados desta audiência, enfatiza-se a decisão da agência em considerar a minigeração como "a central geradora de energia elétrica com potência superior a $75 \mathrm{~kW}$ e menor ou igual a $5 \mathrm{MW}$ e que utilize cogeração qualificada" e a permitir a compensação energética do montante gerado em determinados horários e estações.

Estes progressos permitiram o surgimento de novas modalidades de sistemas de geração distribuída como: os empreendimentos com múltiplas unidades consumidoras que consistem em instalações para uso comum em condomínios e a possibilidade de utilização dos créditos energéticos gerados serem divididos entre os condôminos; geração compartilhada que permite aos diferentes consumidores a possibilidade de associação à cooperativa ou consórcio; autoconsumo remoto que concede aos consumidores a utilização dos créditos energéticos em unidade geradora que não seja a principal.

De acordo com a Associação Brasileira de Energia Solar Fotovoltaica (ABSOLAR), houve aumento de $172 \%$ no número de sistemas instalados no período de 2017 a 2018. Em 2019, a expectativa é que os projetos de geração distribuída adicionem mais de $628 \mathrm{MW}$ de capacidade de geração no país, crescimento de $125 \%$ em relação ao ano anterior.

O aumento das tarifas aplicadas pelas concessionárias simultaneamente à redução dos custos do sistema pode colaborar para a viabilidade econômica dos projetos de forma a manter este crescimento. [2]

\section{Sistema de compensação de energia}

A Resolução Normativa ANEEL n. 482, de abril de 2012, permitiu a possibilidade de incorporação dos sistemas de micro e minigeração no Brasil. O sistema de compensação de energia através do empréstimo gratuito, junto à distribuidora local, de energia ativa injetada na rede para ser deduzida do consumo do consumidor.

O sistema de compensação permite utilizar o excedente da produção de energia posteriormente pelo consumidor. A energia é inserida na rede da distribuidora transformando-se em forma de créditos de energia em $\mathrm{kWh}$.

A norma foi revisada em 2015 através da Resolução Normativa n. 687/2015 que alterou, dentre outros pontos, o período de validade dos créditos de 36 para 60 meses, dos quais também podem ser utilizados em unidades consumidoras de mesmo CPF ou CNPJ localizadas em outro local desde que esteja dentro da área de mesma distribuidora.

A revisão ainda aponta a possibilidade de outras formas de utilizar o sistema de compensação de energia através de consórcios ou cooperativas e condomínios, a geração compartilhada. Ambos são semelhantes na forma de distribuição de créditos entre os seus integrantes. No consórcio ou cooperativa cabe ao mesmo definir o percentual de cada uma das unidades consumidoras, já no condomínio, cabe ao titular escolher a quantidade alocada em cada unidade podendo ser residencial, comercial ou industrial.

\section{Conjuntura atual}

A Bloomberg projeta que $75 \%$ da capacidade instalada no Brasil de geração fotovoltaica seja em geração distribuída em 2040, representando pelo menos $82 \mathrm{GW}$. A cada ano o número de instalações no Brasil aumenta, apesar de barreiras regulatórias, tributárias e de financiamento ainda existentes. [3]

A Resolução Normativa n. 482/2012 é a norma vigente que rege todo o mercado de projetos de geração distribuída no Brasil. Ela estabelece as condições gerais para o acesso de micro e minigeração distribuída aos sistemas de distribuição de energia elétrica, e cria a possibilidade de compensação entre 
a energia elétrica ativa injetada pela unidade gerador-consumidor e a rede distribuidora na qual está conectada. [3]

Em 2015 a REN 482/2012 foi atualizada através da REN 687/2015 modificando alguns pontos importantes como:

- Limite de potência instalada da microgeração de $100 \mathrm{~kW}$ para $75 \mathrm{~kW}$ e de minigeração de $1 \mathrm{MW}$ para $5 \mathrm{MW}$;

- Possibilidade de autoconsumo remoto de múltiplas unidades como condomínios;

- Aumento do prazo de utilização dos créditos de 36 para 60 meses;

- Processo de conexão simplificado: projetos de microgeração tiveram o prazo máximo reduzido de 82 dias para 34 dias

Através dos Procedimentos de Distribuição (PRODIST) a Agencia Nacional de Energia Elétrica (ANEEL) detalha todo o procedimento de solicitação de acesso de projetos de micro e minigeração distribuída que participa do sistema de compensação de energia.

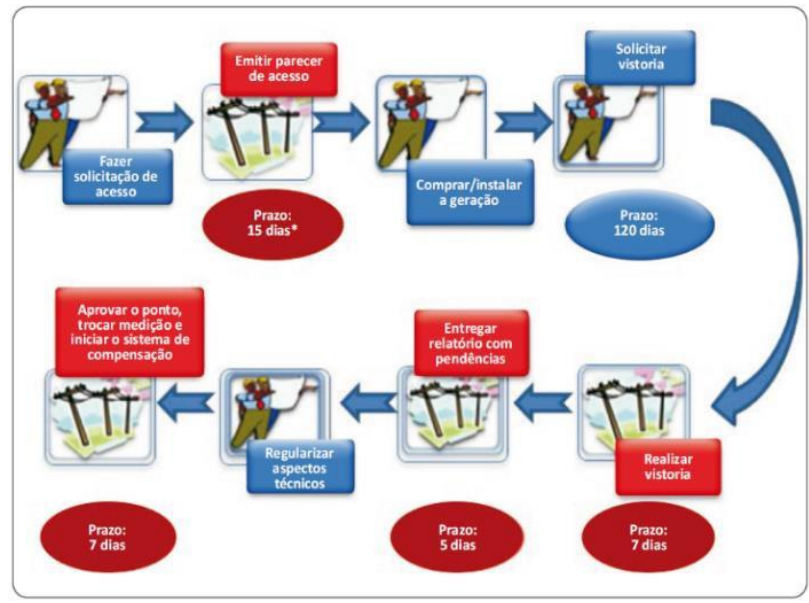

Figura 1: Procedimentos e etapas de acesso. Fonte: Aneel 2016.

\subsection{Tributação}

O Convênio ICMS no $16 / 2015$ concede a isenção do ICMS incidente sobre a energia elétrica fornecida pela distribuidora à unidade consumidora, na quantidade correspondente à energia injetada na rede de distribuição pela mesma unidade consumidora [4]
No país, 24 mais o Distrito Federal aderiu ao convênio e isentam o consumidor da incidência do imposto na energia gerada. Santa Catarina e Paraná, embora já autorizados a conceder a isenção desde julho de 2018, optaram por oferecer o benefício pelo prazo máximo de 48 meses, podendo revogar antes do período. [5]

A Lei no 13.169/15, artigo $8^{\circ}$ (Brasil, 2015a), desde novembro de 2015 , isenta projetos de geração distribuída sob a REN 482 de PIS/PASEP e COFINS na energia elétrica ativa fornecida pela distribuidora na quantidade correspondente à energia injetada mais créditos acumulados pela unidade consumidora. Portanto, a incidência do PIS e COFINS passou a acontecer apenas sobre a diferença positiva entre a energia consumida e a energia injetada pela unidade consumidora com micro ou minigeração distribuída. [3]

\section{Software SOLergo}

O software SOLergo [6], de origem Italiana, foi utilizado para comparar com os resultados obtidos através da ferramenta produzida.

Apesar de ter origem estrangeira, o SOLergo foi adaptado à realidade brasileira. Todos os parâmetros são flexíveis e a utilização de coordenadas geográficas possibilita o cálculo mais próximo possível da realidade do local onde o sistema será instalado.

O programa conta com uma grande base de dados como, climáticos, de painéis solares, inversores e cabos, por exemplo, para iniciar o dimensionamento do sistema de produção de energia. Posteriormente é possível realizar a análise de retorno financeiro.

Portanto, a incidência do PIS e COFINS passou a acontecer apenas sobre a diferença positiva entre a energia consumida e a energia injetada pela unidade consumidora com micro ou minigeração distribuída. [3]

\section{Método}

O método aplicado neste artigo, propõe a utilização de simulação numérica pelo Método de Monte Carlo a fim de analisar o impacto do risco de geração na decisão de implantação do sistema fotovoltaico. 


\subsection{Análise determinística}

A modelagem econômica da vantajosidade de instalação do sistema consiste inicialmente na criação do fluxo de caixa do projeto. O primeiro passo consiste em apurar mensalmente a diferença entre a geração do sistema e o consumo do investidor na forma da Equação 1:

$$
G_{t}\left(1-D_{n}\right)-C_{t}
$$

Em que:

$G_{t}$ : Geração esperada do sistema no mês " $t$ "

$D_{n}$ : Perda acumulada do sistema no ano " $n$ "

$C_{t}$ : Consumo esperado no mês " $t$ "

Caso a Equação 1 seja positiva para dado mês " $t$ ", o saldo a maior será acrescido à geração posterior na forma da Equação 2:

$$
G_{t}\left(1-D_{n}\right)-C_{t}+\text { Saldo }_{t-1}
$$

Em que:

Saldo $_{t-1}$ : Saldo positivo do mês anterior

Caso a Equação 2 seja negativa para dado mês " $t$ ", o investidor arcará com o valor máximo entre: 1 . a aplicação da tarifa atualizada da distribuidora e o montante mínimo de consumo para dada tensão e 2 . a aplicação da tarifa atualizada da distribuidora e o valor efetivamente apurado no mês " $t$ ". O cálculo está demonstrado na Equação 3:

$$
\begin{array}{r}
P_{t}>0 \leftrightarrow\left[G_{t}\left(1-D_{n}\right)-C_{t}+\text { Saldo }_{t-1}\right]<0 \mid P_{t} \\
=\operatorname{Max}\left[T D_{t} . E Q_{2} ; T D_{t} . C_{\text {min }}\right]
\end{array}
$$

Em que:

$P_{t}$ : Pagamento à distribuidora no mês " $t$ " $T D_{t}$ : Tarifa da distribuidora atualizada no mês " $t$ " $E Q_{2}$ : Saldo negativo de energia da Equação 2 $C_{\min }$ : Consumo mínimo para dada tensão

Caso a Equação 2 seja positiva para dado mês " $t$ ", o investidor arcará com o valor mínimo referente a aplicação da tarifa cobrada pela distribuidora e o montante mínimo de consumo para dada tensão na forma da Equação 4:

$$
\begin{gathered}
P_{t}>0 \leftrightarrow\left[G_{t}\left(1-D_{n}\right)-C_{t}+\text { Saldot }_{t-1}\right]>0 \mid P_{t} \\
=T D_{t} . C_{\text {min }}
\end{gathered}
$$

A variável que representa a Tarifa da Distribuidora $\left(T D_{t}\right)$ foi projetada de modo a representar o histórico entre o ano de 2001 e 2018 na forma da Equação 5:

$$
T D_{t}=T D_{0}\left(1+\frac{\sum_{2001}^{2018} \% T D}{18}\right)^{n} \cdot \beta
$$

Em que:

$T D_{0}$ : Tarifa inicial do estudo

$\sum_{2001}^{2018} \%$ T : somatório dos percentuais aplicados à

tarifa da distribuidora entre os anos de 2001 e 2018

$\beta$ : representa o mês em que será aplicado o reajuste

A economia efetivamente apurada pelo investidor no mês " $t$ " será a diferença entre o pagamento à distribuidora nos cenários sem e com a instalação do sistema fotovoltaico na forma da Equação 6:

\begin{tabular}{|c|c|}
\hline Item & $+/-$ \\
\hline a. Geração & $(+)$ \\
\hline b. Consumo & $(-)$ \\
\hline c. Saldo & $(=a-b)$ \\
\hline d. $P_{t}$ & $(-)$ \\
\hline e. Economia do sistema & $(+)$ \\
\hline f. Despesas O\&M & $(-)$ \\
\hline g. Despesas Inversores & $(-)$ \\
\hline h. Investimento & $(-)$ \\
\hline Fluxo resultante & $-(h+g+f)+e$ \\
\hline
\end{tabular}

$$
C_{t}\left(T D_{t}\right)-P_{t}
$$

Para composição do Fluxo de Caixa Nominal foram consideradas despesas com Operação e Manutenção, Troca de Inversores e Investimento Total.

Tabela 1: Sazonalização da incidência solar. Fonte: Autores

Fonte: Dados dos autores

O Fluxo de Caixa Real (descontado pela inflação) foi obtido pelo desconto do Fluxo de Caixa Nominal pela aplicação de taxa de inflação esperada. Após a 
obtenção do Fluxo de Caixa Real, aplicou-se o Valor Presente Líquido descontado por determinada taxa de desconto na forma da Equação 7:

$$
V P L=F C_{0}+\sum_{t}^{T} \underset{(1+k)^{t}}{F C_{\underline{t}}}
$$

Em que:

$F C_{0}$ : Fluxo de Caixa no período inicial

$F C_{t}$ : Fluxo de Caixa no período " $t$ "

$\mathrm{k}$ : Taxa de desconto

\subsection{Análise Probabilística}

A aleatoriedade da incidência solar foi representada pela função de distribuição de probabilidade de Weibull que explica a variação na produtividade horária mensal média $(\mathrm{kWh})$ do sistema em determinado ano.

Desta forma, o termo de aleatoriedade foi adicionado à Equação 2 na forma da Equação 8:

$$
\left(1-\exp \left[-\left(\frac{{ }^{X}}{\eta}\right)^{\beta}\right) \cdot(1-D)_{n}-C+{ }_{t} \text { Saldo } \quad{ }_{t-1}\right.
$$

Em que:

$\eta$ : Parâmetro de escala

$\beta$ : Parâmetro de forma

\subsection{Benchmark de mercado e tomada de decisão}

O Valor Presente Líquido do projeto foi comparado com o Fluxo de Caixa de Título Público do Governo Federal de duração compatível com o tempo de análise do projeto. A referência (benchmark) de mercado escolhida foi o Tesouro IPCA+ com Juros Semestrais (NTN-B) e vencimento em 15/05/2045.

Investimentos em títulos públicos são considerados seguros. Títulos Tesouro IPCA+ garantem ao investidor proteção contra a inflação e manutenção do poder de compra. O rendimento é composto por duas parcelas (IPCA + spread).

O pagamento de Juros Semestrais garante ao investidor o retorno do seu investimento ao longo do período de aplicação. Na data de vencimento do título, o valor é resgatado atualizado pela inflação e acrescido do último pagamento de juros semestrais.

A tomada de decisão será analisada como vantajosa se o valor presente líquido gerado pela instalação do sistema fotovoltaico for maior do que aquele produzido pelo benchmark de mercado dada a mesma taxa de desconto.

\section{Premissas}

As principais premissas utilizadas neste estudo estão demonstradas nos Quadros 1 a 7. 0 Quadro 1 a seguir demonstra informações financeiras e taxa de deterioração anual do sistema solar.

Quadro 1: Dados para análise.

\begin{tabular}{|l|r|c|}
\hline \multicolumn{3}{|c|}{ Dados para análise } \\
\hline Data de Início & \multicolumn{2}{|c|}{$01 / 09 / 2019$} \\
\hline Potência do Sistema & 16,08 & $\mathrm{kWp}$ \\
\hline Investimento & $93.399,55$ & $\mathrm{R} \$$ \\
\hline Consumo Anual & $25.157,70$ & $\mathrm{kWh}$ \\
\hline Inflação & 4,50 & $\%$ \\
\hline Taxa de Desconto & 6,40 & $\%$ \\
\hline Taxa Média de Reajuste da Tarifa & 9,24 & $\%$ \\
\hline Deterioração anual do sistema & 0,80 & $\%$ \\
\hline Despesa O\&M (\% Investimento) & 1,00 & $\%$ \\
\hline Despesa Inversores (10 e 20 anos) & $20.000,00$ & $\mathrm{R} \$$ \\
\hline
\end{tabular}

Fonte: Dados dos autores

O Quadro 2 apresenta a sazonalidade climática no período de 12 meses de acordo com o Centro de Referência para Energia Solar e Eólica (CRESESB).

\section{Quadro 2: Dados Climáticos}

\begin{tabular}{|c|c|}
\hline Mês & (kWh/ m².dia) \\
\hline Janeiro & 5,75 \\
\hline Fevereiro & 5,71 \\
\hline Março & 5,71 \\
\hline Abril & 5,09 \\
\hline Maio & 4,43 \\
\hline Junho & 4,13 \\
\hline Julho & 4,18 \\
\hline Agosto & 4,93 \\
\hline Setembro & 5,51 \\
\hline Outubro & 5,71 \\
\hline Novembro & 5,92 \\
\hline Dezembro & 5,84 \\
\hline Fonte: Cresesb
\end{tabular}

Fonte: Cresesb 
O Quadro 3 apresenta a sazonalidade de consumo da unidade no período de 12 meses.

Quadro 3: Consumo da unidade

\begin{tabular}{|c|c|}
\hline Mês & Consumo \\
\hline Janeiro & 2.126 \\
\hline Fevereiro & 2.271 \\
\hline Março & 2.092 \\
\hline Abril & 2.087 \\
\hline Maio & 2.148 \\
\hline Junho & 2.220 \\
\hline Julho & 2.072 \\
\hline Agosto & 1.922 \\
\hline Setembro & 2.001 \\
\hline Outubro & 2.105 \\
\hline Novembro & 2.104 \\
\hline Dezembro & 2.009 \\
\hline Total & $\mathbf{2 5 . 1 5 7}$ \\
\hline
\end{tabular}

Fonte: Dados dos autores

Os Quadro 4 e 5 apresentam a produção prevista de energia, em kWh, entre os meses de Janeiro a Junho e Julho a Dezembro. É possível notar que não há incidência de irradiação solar por aproximadamente 12 horas. Nestecaso, não há geração de energia pelo sistema.

Quadro 4: Produção prevista de energia em kWh entre Janeiro e Junho.

\begin{tabular}{|c|r|r|r|r|r|r|}
\hline Hora & \multicolumn{1}{|l}{ Jan } & \multicolumn{1}{l}{ Fev } & \multicolumn{1}{l}{ Mar } & \multicolumn{2}{l}{ Mai } & \multicolumn{1}{l|}{ Jun } \\
\hline $\mathbf{0 - 4}$ & 0 & 0 & 0 & 0 & 0 & 0 \\
\hline $\mathbf{5}$ & 0,1 & 0,1 & 0 & 0 & 0 & 0 \\
\hline $\mathbf{6}$ & 1,7 & 1,5 & 1,3 & 0,9 & 0,6 & 0,5 \\
\hline $\mathbf{7}$ & 3,8 & 3,6 & 3,4 & 2,8 & 2,3 & 2,1 \\
\hline $\mathbf{8}$ & 6 & 5,8 & 5,7 & 4,9 & 4,1 & 3,8 \\
\hline $\mathbf{9}$ & 8 & 7,9 & 7,8 & 6,8 & 5,9 & 5,4 \\
\hline $\mathbf{1 0}$ & 9,5 & 9,4 & 9,3 & 8,3 & 7,2 & 6,6 \\
\hline $\mathbf{1 1}$ & 10 & 10 & 10 & 9 & 7,8 & 7,2 \\
\hline $\mathbf{1 2}$ & 10 & 10 & 9,9 & 8,8 & 7,7 & 7,1 \\
\hline $\mathbf{1 3}$ & 9 & 8,9 & 8,8 & 7,8 & 6,8 & 6,3 \\
\hline $\mathbf{1 4}$ & 7,3 & 7,2 & 7,1 & 6,2 & 5,3 & 4,9 \\
\hline $\mathbf{1 5}$ & 5,2 & 5,1 & 4,9 & 4,2 & 3,5 & 3,2 \\
\hline $\mathbf{1 6}$ & 3,1 & 2,9 & 2,6 & 2,1 & 1,7 & 1,5 \\
\hline $\mathbf{1 7}$ & 1,1 & 0,9 & 0,7 & 0,5 & 0,3 & 0,3 \\
\hline $\mathbf{1 8 - 2 3}$ & 0 & 0 & 0 & 0 & 0 & 0 \\
\hline
\end{tabular}

Fonte: Software SOLergo
Quadro 5: Produção prevista de energia em kWh entre Julho e Dezembro.

\begin{tabular}{|c|c|c|c|c|c|c|}
\hline Hora & Jul & Ago & Set & Out & Nov & Dez \\
\hline $0-4$ & 0 & 0 & 0 & 0 & 0 & 0 \\
\hline 5 & 0 & 0 & 0 & 0,05 & 0,1 & 0,14 \\
\hline 6 & 0,52 & 0,78 & 1,13 & 1,41 & 1,68 & 1,74 \\
\hline 7 & 2,11 & 2,66 & 3,2 & 3,53 & 3,85 & 3,87 \\
\hline 8 & 3,87 & 4,69 & 5,42 & 5,79 & 6,14 & 6,11 \\
\hline 9 & 5,5 & 6,57 & 7,46 & 7,84 & 8,22 & 8,13 \\
\hline 10 & 6,73 & 7,98 & 8,97 & 9,37 & 9,75 & 9,63 \\
\hline 11 & 7,33 & 8,66 & 9,71 & 10,1 & 10,5 & 10,4 \\
\hline 12 & 7,2 & 8,5 & 9,54 & 9,94 & 10,3 & 10,2 \\
\hline 13 & 6,36 & 7,53 & 8,49 & 8,9 & 9,28 & 9,18 \\
\hline 14 & 4,97 & 5,92 & 6,75 & 7,15 & 7,53 & 7,47 \\
\hline 15 & 3,28 & 3,96 & 4,62 & 5,01 & 5,35 & 5,35 \\
\hline 16 & 1,58 & 1,97 & 2,44 & 2,79 & 3,1 & 3,15 \\
\hline 17 & 0,27 & 0,37 & 0,57 & 0,82 & 1,06 & 1,14 \\
\hline 18 & 0 & 0 & 0 & 0 & 0,01 & 0,02 \\
\hline $\begin{array}{c}19- \\
23\end{array}$ & 0 & 0 & 0 & 0 & 0 & 0 \\
\hline
\end{tabular}

Fonte: Software SOLergo

O Quadro 6 sintetiza mensalmente as informações dos Quadros 4 e 5.

Quadro 6: Produção total prevista de energia em kWh.

\begin{tabular}{|c|r|}
\hline Mês & Produção \\
\hline Jan & $2.325,00$ \\
\hline Fev & $2.048,48$ \\
\hline Mar & $2.218,67$ \\
\hline Abr & $1.865,70$ \\
\hline Mai & $1.644,86$ \\
\hline Jun & $1.463,70$ \\
\hline Jul & $1.541,32$ \\
\hline Ago & $1.847,29$ \\
\hline Set & $2.049,00$ \\
\hline Out & $2.254,01$ \\
\hline Nov & $2.307,00$ \\
\hline Dez & $2.371,19$ \\
\hline Total & $\mathbf{2 3 . 9 3 6 , 2 2}$ \\
\hline
\end{tabular}

A evolução do reajuste da tarifa da distribuidora CELPE está demonstrada, desde 2001, no Quadro 7. A média aritmética destes valores $(9,24 \%)$ foi utilizada como projeção neste estudo. 
Quadro 7: Evolução do reajuste da tarifa da distribuidora.

\begin{tabular}{|l|l|c|}
\hline Ano & Legislação & Reajuste \\
\hline 2001 & Resolução $93 / 2001$ & $14,85 \%$ \\
\hline 2002 & Resolução 152/2002 & $15,12 \%$ \\
\hline 2003 & Resolução 127/2003 & $28,47 \%$ \\
\hline 2004 & Resolução 60/2004 & $11,42 \%$ \\
\hline 2005 & Resolução 112/2005 & $24,43 \%$ \\
\hline 2006 & Resolução 327/2006 & $10,66 \%$ \\
\hline 2007 & Resolução 459/2007 & $2,45 \%$ \\
\hline 2008 & Resolução 642/2008 & $3,34 \%$ \\
\hline 2009 & Resolução 815/2009 & $-6,24 \%$ \\
\hline 2010 & Resolução 973/2010 & $-8,70 \%$ \\
\hline 2011 & Resolução $1143 / 2011$ & $8,27 \%$ \\
\hline 2012 & Resolução 1283/2012 & $5,41 \%$ \\
\hline 2013 & Resolução 1519/2013 & $1,32 \%$ \\
\hline 2014 & Resolução $1723 / 2014$ & $17,75 \%$ \\
\hline 2015 & Resolução $1885 / 2015$ & $11,25 \%$ \\
\hline 2016 & Resolução 2067/2016 & $9,99 \%$ \\
\hline 2017 & Resolução 2226/2017 & $7,62 \%$ \\
\hline 2018 & Resolução 2388/2018 & $8,89 \%$ \\
\hline
\end{tabular}

Fonte: Aneel

\section{Resultados e Análise}

A taxa de desconto $(6,40 \%$ a.a. $)$ utilizada representa a atual Taxa de CDI (Certificado de Depósitos Interbancários). O CDI é benchmark para diversos produtos financeiros e tende a acompanhar o comportamento da SELIC (Sistema Especial de Liquidação e Custódia) que representa a taxa básica de juros da economia.

A utilização do CDI como taxa de desconto almeja comparar os ganhos econômicos decorrentes da instalação do sistema fotovoltaico na unidade consumidora com os ganhos econômicos gerados por produtos financeiros disponíveis a quaisquer investidores.

Desta forma, este estudo considera que o CDI representa o custo de oportunidade de qualquer investidor sob um cenário conservador. O VPL é muito sensível à definição da taxa de desconto e a utilização de uma taxa indevida pode enviesar os resultados.

O Quadro 8 apresenta os valores de TIR (Taxa Interna de Retorno) e VPL com base nas premissas apresentadas.
Quadro 8: Comparativo de Fluxos

\begin{tabular}{|c|c|c|c|}
\hline Investimento & VPL1 & TIR Real & Payback \\
\hline Solar & 493,29 & $24,54 \%$ & 2024 \\
\hline NTN-B 2045 & 13,25 & $2,63 \%$ & 2045 \\
\hline
\end{tabular}

A estimativa de VPL pela decisão de instalar o sistema fotovoltaico na unidade consumidora é de aproximadamente $\mathrm{R} \$ 493,29$ mil. Isto significa que, caso o investidor almeje aplicar o capital inicial ( $R$ 93,39 mil) no CDI e receber 6,40\% a.a., a alternativa de instalação do sistema fotovoltaico gerará $\mathrm{R} \$$ 493,29 mil já descontado o investimento inicial.

O Tesouro IPCA+ 2045 apresentou apenas a geração de VPL de R\$13,25 mil descontados pelo CDI. O Payback deste investimento ocorre apenas em 2045 devido à modalidade de pagamento de Juros Semestrais ao longo do tempo e do principal reajustado apenas no vencimento.

O Payback encontrado para o sistema solar foi no ano de 2024, entre 6 e 7 anos do investimento inicial. A TIR representa um parâmetro de custo de oportunidade. Neste caso, o sistema solar só não será vantajoso caso alguma outra alternativa seja capaz entregar pelo menos $25 \%$ de retorno anual real (acima da inflação).

Não há, no mercado financeiro, produto financeiro capaz de entregar rentabilidade real neste patamar sem que seja necessário que o investidor assuma considerável parcela de risco.

A Figura 2 representa o comparativo entre os fluxos reais (descontados pela inflação projetada) entre o investimento em títulos públicos IPCA+ 2045 e sistema fotovoltaico. Dado que a tarifa de reajuste assumida para a distribuidora é maior do que a inflação, o ganho econômico de instalação do sistema é crescente.

Dentre os anos analisados, o sistema solar apresenta queda no fluxo em dois pontos (2028 e 2038) devido à substituição dos inversores. O valor final maior do título público, em 2045, representa o resgate do principal atualizado.

Mesmo com a deterioração do sistema à taxa de $0,80 \%$ ao ano, o valor reajustado a ser pago pela distribuidora avança mais que a inflação e contribui para os ganhos econômicos do fluxo do cenário de instalação do sistema. 


\section{VPL NTN-B $2045 \times$ S Sistema Fotovoltaico}

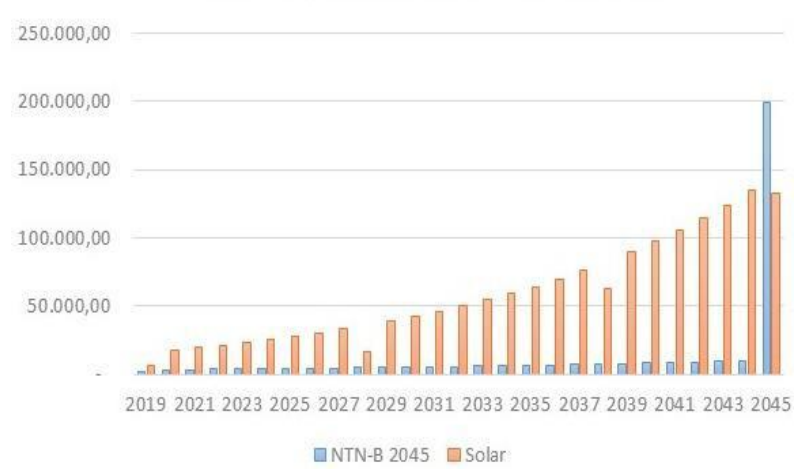

Figura 2: Comparativo entre fluxos reais Fonte: Cálculo dos autores

A decisão de investimento no sistema solar é função direta das variáveis de pressuposto (premissas) utilizadas. A Figura 3 apresenta o impacto, em termos de contribuição à variação percentual do VPL, de variações de $10 \%$ para mais e para menos de determinadas premissas.

Desta forma, os valores apresentados na Figura 3 representam a variação do VPL Real do ganho econômico como função da variação uniforme de $+10 \%$ e $-10 \%$ de cada premissa.

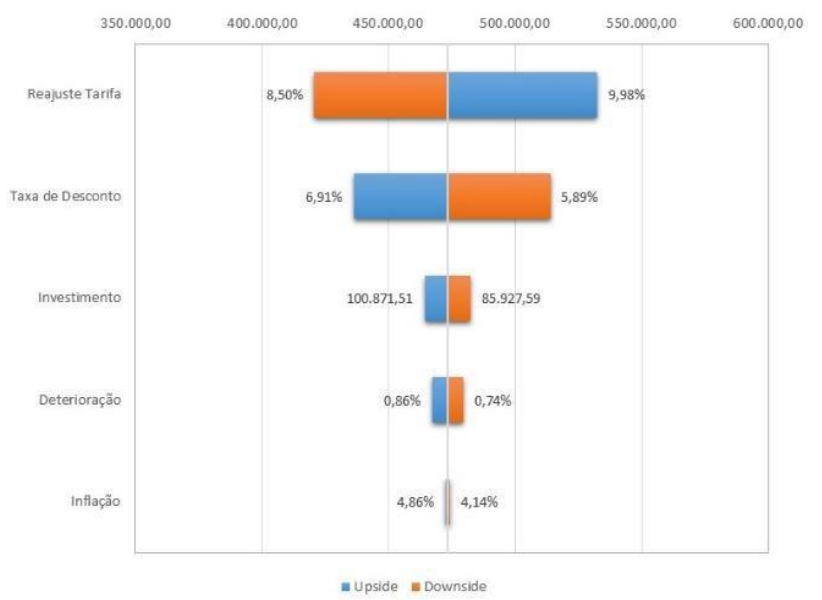

Figura 3: Impacto das premissas no VPL Fonte: Cálculo dos autores

O Quadro 9 apresenta em números os resultados da Figura 3.
Quadro 9: Desvantagem, Vantagem e Intervalo

\begin{tabular}{|l|rrr|r|rrr|}
\hline \multirow{2}{*}{ Pressuposto } & \multicolumn{3}{|c|}{ VPL Real (R\$ Milhões) } & \multirow{2}{*}{ VE } & \multicolumn{3}{|c|}{ Insumo } \\
\cline { 2 - 4 } \cline { 7 - 8 } & \multicolumn{1}{c|}{$\mathbf{D}$} & $\mathbf{V}$ & $\mathbf{1}$ & & $\mathbf{D}$ & $\mathbf{V}$ & $\mathbf{C B}$ \\
\hline Reajuste Tarifa & 420,60 & 532,33 & 111,73 & $65,78 \%$ & $8,50 \%$ & $9,98 \%$ & $9,24 \%$ \\
Taxa de Desconto & 513,88 & 436,30 & 77,58 & $97,49 \%$ & $5,89 \%$ & $6,91 \%$ & $6,40 \%$ \\
Investimento & 482,30 & 464,28 & 18,02 & $99,20 \%$ & 85,93 & 100,87 & 93,40 \\
Deterioração & 479,39 & 467,18 & 12,21 & $99,99 \%$ & $0,74 \%$ & $0,86 \%$ & $0,80 \%$ \\
Inflação & 474,10 & 472,44 & 1,65 & $100,00 \%$ & $4,14 \%$ & $4,86 \%$ & $4,50 \%$ \\
\hline
\end{tabular}

D: Desvantagem, valor mínimo

V: Vantagem, valor máximo

I: Intervalo

VE: Variação Explicada acumulada

CB: Caso base

Fonte: Cálculo dos autores

Os valores demonstram que variações na variável "Reajuste Tarifa" de $10 \%$ a menor e a maior contribuem para variações no VPL Real do ganho econômico entre $R \$ 420,60$ mil e $R \$ 532,33$ mil. $O$ poder de explicação desta variável frente às escolhidas para a análise é de $65,78 \%$.

A variável "Taxa de Desconto", em seu valor mínimo, produz VPL Real de $\mathrm{R} \$ 513,88$ mil. Isto significa que quanto menor a taxa de desconto maior será o VPL. O poder de explicação desta variável na decisão de instalação do sistema é de $31,71 \%$. As demais variáveis (Investimento, Deterioração e Inflação) não apresentaram resultados significativos.

Os dados demonstram que a decisão de instalação do sistema solar depende principalmente dos futuros índices de reajuste da distribuidora e do custo de oportunidade do investidor.

Com a finalidade de analisar o comportamento do VPL Real do ganho econômico frente a múltiplos cenários de reajuste de tarifa $(4,5 \%$ a $9,50 \% \mathrm{em}$ passos de $1 \%$ ), as Figuras 4 e 5 apresentam a análise destes cenários sob a premissa de aleatoriedade da irradiação solar conforme proposto na Equação 8.

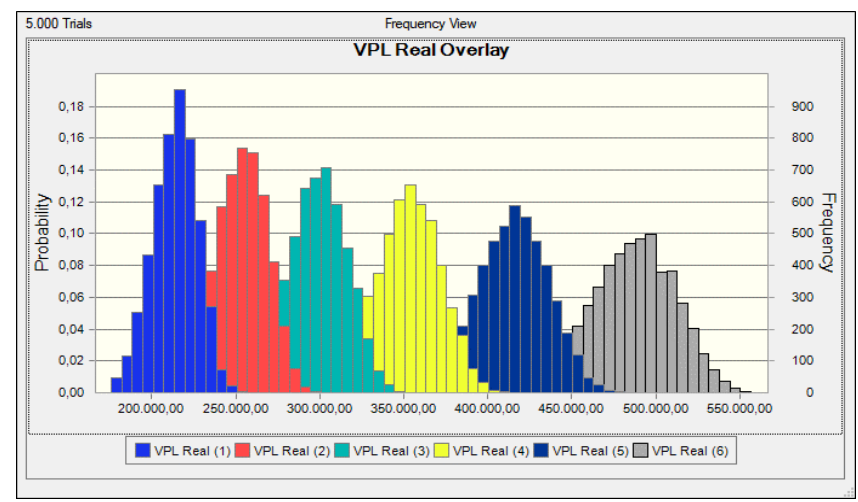

Figura 4: Análise de risco - Distribuições Fonte: Cálculo dos autores 


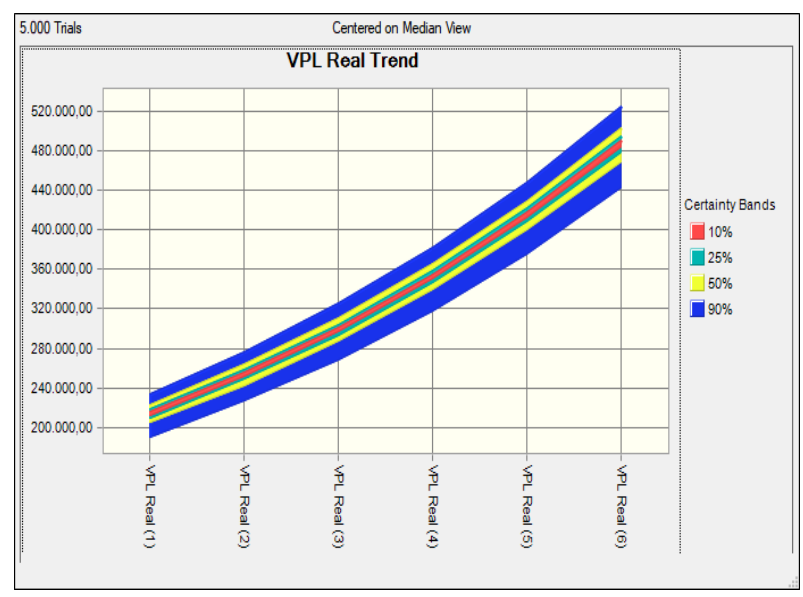

Figura 5: Análise de risco - Tendências Fonte: Cálculo dos autores

A Figura 4 apresenta a distribuição de probabilidade do VPL Real do ganho econômico em relação ao reajuste de $4,5 \%$ da tarifa da distribuidora (valor igual à inflação projetada).

Mesmo sob este cenário conservador, a distribuição de probabilidade resultante retorna valores positivos (primeiro quadrante). Isto significa que a probabilidade de perda econômica com a instalação do sistema é nula mesmo sob a premissa de reajuste da tarifa da distribuidora de $4,5 \%$ a.a. e o VPL do ganho econômico mínimo é de aproximadamente $\mathrm{R} \$ 175$ mil neste cenário.

Os valores das distribuições de probabilidade deslocam-se para a direita à medida que o percentual de reajuste aumenta. $O$ desvio-padrão das distribuições também aumenta devido ao maior impacto da deterioração do sistema e da aleatoriedade da irradiação solar no VPL Real do benefício econômico.

A Figura 5 representa de forma similar os valores das distribuições da Figura 4. A faixa azul representa $90 \%$ de certeza de que o VPL Real do benefício econômico estará situado entre o máximo e o mínimo. Esta faixa engloba todas as outras faixas.

\section{Conclusão}

A instalação do sistema solar para esta unidade consumidora demonstrou-se economicamente vantajosa com base nas premissas adotadas e gerou valor presente líquido aproximadamente 40 vezes maior do que um investimento conservador em títulos públicos.

Todavia, caso o consumidor ou empreendedor consiga rentabilizar o seu capital à taxa maior do que $25 \%$ ao ano acima da inflação, a decisão de instalação do sistema solar requer cautela.

A migração para energia solar funciona como uma proteção (hedge) aos aumentos históricos sucessivos nas tarifas das distribuidoras. Quanto maior for 0 aumento esperado pelo investidor para estas tarifas, mais vantajoso será a instalação do sistema solar ao nível atual de investimento.

\section{Referências}

[1] ZILLES, Roberto et al. Sistemas fotovoltaicos conectados à rede elétrica. 2016.

[2] ABSOLAR. Energia solar deve crescer $44 \%$ no Brasil em 2019 com impulso da geração distribuída.

<http://www.absolar.org.br/noticia/noticiasexternas/energia-solar-deve-crescer-44-no- brasil-em2019-com-impulso-de-geracao- distribuida.html>. Acesso em: 24 jan. 2019.

[3] SEBRAE. Cadeia de valor da energia solar fotovoltaica no Brasil. 2017.

[4] DIÁRIO OFICIAL DA UNIÃO. Convênio ICMS 16/2015. 2015.

<https://www.confaz.fazenda.gov.br/legislacao/c onvenios/2015/cv016_15>. Acesso em: 09 mai. 2019.

[5] BLUESOL. Isenção de ICMS para energia solar. <https://blog.bluesol.com.br/infograficoisencao-de-icms-para-energia-solar $>$. Acesso em: 09 mai. 2019.

[6] SOLergo. Software disponível em <https://www.electrographics.com.br/produtos/s olergo >. Acesso em: 11 abr. 2019 